\title{
Supramolecular Dynamics of Thalidomide and its Derivatives in Water-Sediment System
}

\author{
IMRAN ALI, ${ }^{1 *}$ VINOD K. GUPTA, ${ }^{2}$ AND HASSAN Y. ABOUL-ENEIN ${ }^{3,4 *}$ \\ ${ }^{1}$ Department of Chemistry, Jamia Millia Islamia (Central University), New Delhi, India \\ ${ }^{2}$ Department of Chemistry, College of Science, King Fahd University of Petroleum and Minerals, \\ Dhahran - 31261, Kingdom of Saudi Arabia \\ ${ }^{3}$ Department of Pharmaceutical and Medicinal Chemistry, Pharmaceutical and Drug Industries Research Division, \\ National Research Centre, Dokki, Cairo 12311, Egypt \\ ${ }^{4}$ Department of Chemistry, Universiti Teknologi Malaysia (UTM), Skudai. 81310, Johor Darul Ta'zim, Malaysia
}

\begin{abstract}
The contamination of drug residues, including chiral ones, is not acceptable in earth's ecosystem. The dynamicity of enantiomers of thalidomide and its derivatives (3-methyl thalidomide, 3-ethyl thalidomide, and 3-butyl thalidomide) was ascertained at supramolecular level in water-sediment system using solid phase extraction (SPE) and stereoselective HPLC. Enantiomeric separation of these drugs was carried out on Ceramosphere RU-2 $(25 \mathrm{~cm} \times 0.46 \mathrm{~cm}$, particle size $50 \mu \mathrm{m})$ chiral column using pure ethanol $(1.0 \mathrm{ml} / \mathrm{min})$ as eluent at $230 \mathrm{~nm}$ detection. Retention times, capacity, separation, and resolution factors of the enantiomers of these drugs were in the range of $20.0-36.0,2.08-3.93,1.35-1.57$, and $1.0-2.0 \mathrm{~min}$, respectively. Percentage recoveries of the enantiomers in SPE were in the range of 90.0 to 95.0 in water-sediment system. Langmuir and Freundlich model were best fitted for dynamic equilibrium concentrations at different experimental parameters. Thalidomide and its derivatives follow first-order kinetics at dynamic equilibrium. The rate constants of chiral interconversions were 0.390 and 0.385 days $^{-1}$ for $S$ - and $R$-enantiomers, respectively. The uptake of thalidomide by sediment is quite good and of endothermic nature indicating good self-purification capacity of the nature for such toxic species. Chirality 00:000-000, 2009. ๑) 2009 Wiley-Liss, Inc.
\end{abstract}

KEY WORDS: chiral separations; supramolecular chiral dynamics; thalidomide; watersediment system

\section{INTRODUCTION}

Thalidomide [ $\alpha$-( $N$-phthalimido)-glutarimide] is a sedative, hypnotic, and anti-inflammatory medication, which was sold from 1957 to 1961 in almost all European countries under different trade names. But it was banned in 1961 after a calamity called Thalidomide tragedy ${ }^{1}$; because of its teratogenic nature of malformations, including phocomelia, in about 12,000 newly born babies of women in 46 different European countries, who had taken thalidomide during their pregnancies. ${ }^{2}$ Later researchers concluded that this tragedy was due to $S$-(-)-enantiomer of thalidomide. ${ }^{3}$ Basically, thalidomide $[\alpha-(N$-phthalimido)glutarimide] (Fig. 1A) consists of a two ringed structure with an asymmetric carbon in the glutarimide ring, which exists as an equal mixture of $S-(-)$ and $R-(+)$ enantiomers. Recently, thalidomide is used for the treatment of various diseases such as rheumatoid arthritis, graft-versushost, Behcet's syndrome, cutaneous lupus, cancer, and refractory aphthous ulcerations in patients with AIDS in various countries. ${ }^{4-9}$ In 1998, thalidomide has been approved specifically for treating erythema nodosum leprosum (ENL) in leprosy (Hansen's Disease) in USA ${ }^{10}$ and on investigational basis for the treatment of above-cited disease in some North American countries. ${ }^{11-14}$ It has (c) 2009 Wiley-Liss, Inc. been observed that thalidomide showed teratogenic effect even after the administration of safe $R-(+)$-enantiomer because of its rapid conversion into toxic $S$-(-)-form. Some studies have been carried out in vitro on interconversion and stereoselectivity of thalidomide. Besides, in vitro pharmacokinetics of thalidomide have also been established in human beings. ${ }^{15-22}$

In natural conditions, thalidomide is degraded to more than 20 byproducts because of rapid $\mathrm{pH}-$ dependent hydrolysis in aqueous solution. The plasma concentration of thalidomide is $10-15 \mathrm{~h}$ depending on the age, race, and sex of the patients. After this period, thalidomide and its metabolites are excreted to the environment through urine and feces. Therefore, there are good chances of contamination of our natural water resources due to teratogenic thalidomide and its derivatives, which may enter into the

\footnotetext{
*Correspondence to: Hassan Y. Aboul-Enein, Pharmaceutical and Medicinal Chemistry Department, National Research Centre, Dokki, Cairo 12311, Egypt. E-mail: enein@gawab.com or Imran Ali, Department of Chemistry, Jamia Millia Islamia (Central University), New Delhi 110025, India. E-mail: drimran_ali@yahoo.com

Received for publication 11 February 2009; Accepted 27 May 2009

DOI: 10.1002/chir.20757

Published online in Wiley InterScience

(www.interscience.wiley.com).
} 

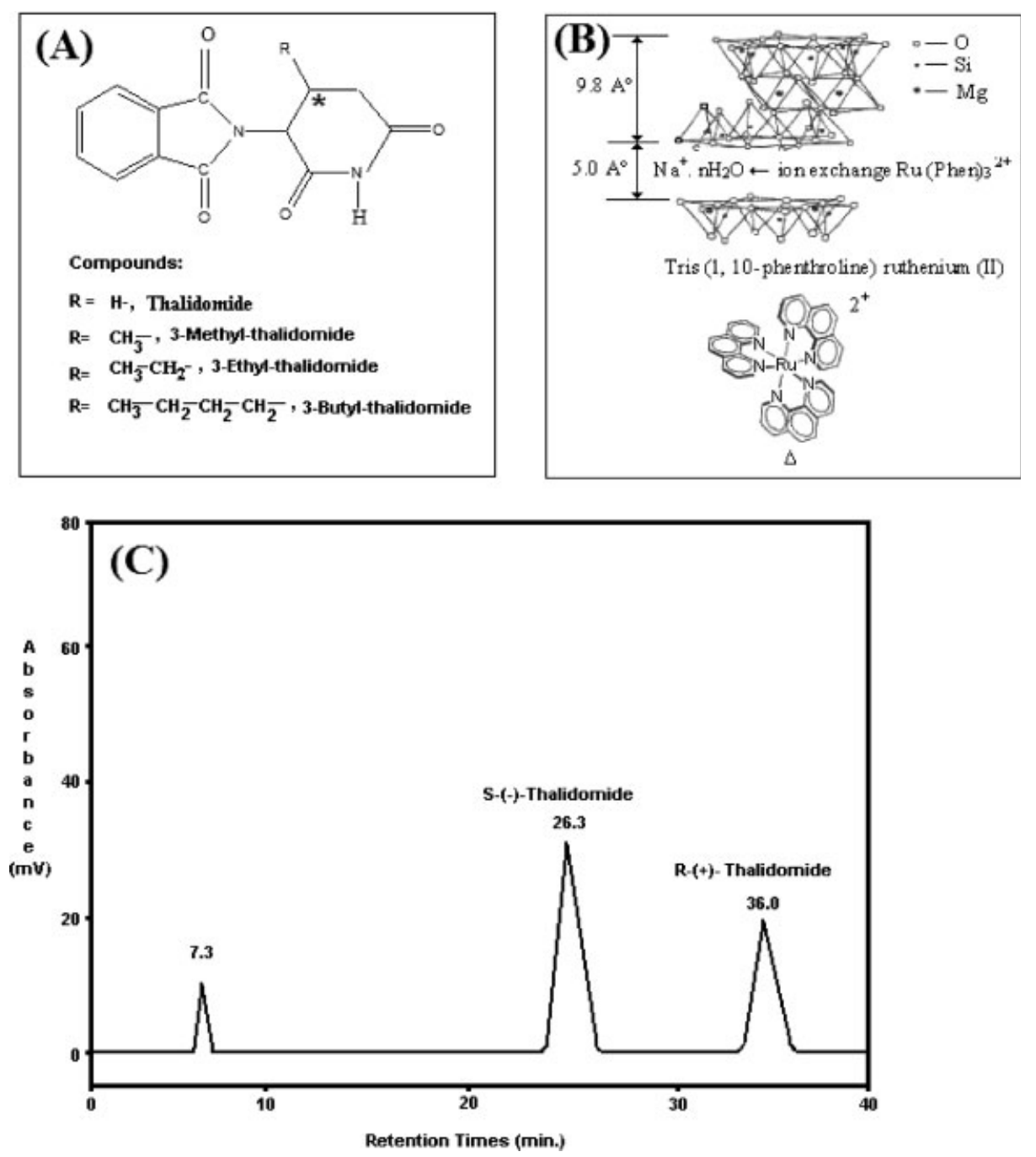

Fig. 1. Structures of (A) thalidomide and its derivatives, (B) Ceramosphere RU-2 chiral stationary phase based on a sodium magnesium silicate, and (C) chiral separation of racemic thalidomide.

human body resulting in serious side effects. In view of this, the study of the fate and dynamics of chiral thalidomide and its derivatives in water and the sediment of rivers, the most probable targets of contamination, are important and essential. The article investigates the dynamics of chiral thalidomide and its derivatives in water and sediment samples at supramolecular level and the results are presented in this article.

\section{EXPERIMENTAL \\ Chemicals and Reagents}

The racemic mixtures and optically active $R-(+)$ - and $S$ $(-)$-forms of thalidomide and its derivatives (3-methyl thalidomide, 3-ethyl thalidomide, and 3-butyl thalidomide) were obtained from Ciba-Geigy (Basle, Switzerland). The solutions $(0.1 \mathrm{mg} / \mathrm{ml})$ were prepared in methanol. Methanol, ethanol, ethyl acetate, acetone, diethylether, chloroform, hexane, and dichloromethane of HPLC grade were purchased from Fisher Scientific (Fairlawn, New Jersey). TriChirality DOI 10.1002/chir fluoroacetic acid, triethylamine, sodiumdihydrogen phosphate, and phosphoric acids were of AR grade and were purchased from Sigma Chemical, USA. Purified water was prepared using a Millipore Milli-Q (Bedford, MA) water purification system. $\mathrm{pH}$ meter of Hach, USA, was used to measure $\mathrm{pH}$ of the solutions. $\mathrm{C}_{18}$ Sep-Pak Vac $(1 \mathrm{cc})$ cartridge was obtained from Waters, USA. The vacuum was generated by the homemade vacuum pump assembly. Zymark Turbo Vap LV evaporator was obtained from Hopkinton, USA. The chiral column Ceramosphere RU-2 $(25 \mathrm{~cm} \times 0.46$ $\mathrm{cm}$, particle size $50 \mu \mathrm{m}$ ) was obtained from Shiseido (Tokyo, Japan). The determination of thalidomide and its derivatives was carried out by stereoselctive HPLC.

\section{Chromatographic Conditions}

An aliquot of $10 \mu$ of each solution was injected on to a HPLC system consisting of Waters solvent delivery pump (model 510), Waters injector (model WISP 710B), Waters tunable absorbance detector (model 484), and Waters integrator (model 740). The order of elution of the enantio- 
mers was confirmed by using optically active $R$-(+)-form of each compounds. The column used was Ceramosphere RU-2 $(25 \mathrm{~cm} \times 0.46 \mathrm{~cm}$, particle size $50 \mu \mathrm{m})$ [Sodium magnesium silicate with optically active (1,10-phenanthroline) ruthenium II complex, $\Delta$ configuration, Fig. 1B]. The mobile phase was ethanol, which was filtered and degassed before use. The flow rates of the mobile phase were $1.0 \mathrm{ml} / \mathrm{min}$ with detection at $230 \mathrm{~nm}$. The chart speed was kept constant at $0.1 \mathrm{~cm} / \mathrm{min}$. All the experiments were carried out at $23^{\circ} \mathrm{C} \pm 1{ }^{\circ} \mathrm{C}$. The chromatographic parameters such as capacity factor $(k)$, separation factor $(\alpha)$, and resolution factor (Rs) were calculated.

\section{Solid Phase Extraction}

Solid phase extraction (SPE) methodology was developed by spiking $10 \mathrm{ml}$ of racemic and optically pure thalidomides and its derivatives $(0.1 \mathrm{mg} / \mathrm{l})$ separately to $90 \mathrm{ml}$ riverine waters; already filtered through Whatman filter papers No. 24. The spiked water samples were shaken for about $5 \mathrm{~min}$ and $\mathrm{pH}$ was adjusted to 7.0 with concentrated sodium hydroxide. The spiked water samples were kept at room temperature overnight. $\mathrm{C}_{18}$ cartridges were preconditioned using methanol $(1.0 \mathrm{ml})$ followed by water $(1.0 \mathrm{ml})$. After equilibration, $100 \mathrm{ml}$ of the spiked water samples were passed through these cartridges at $0.5 \mathrm{ml} / \mathrm{min}$ flow rate. Cartridges were washed with $2.0 \mathrm{ml}$ of deionized water and then dried under vacuum for $5.0 \mathrm{~min}$. Racemic and optically pure thalidomides and its derivatives were eluted from cartridges by using $5.0 \mathrm{ml}$ acetonitrile at $0.5 \mathrm{ml} / \mathrm{min}$ flow rate. Acetonitrile extracts were dried under air at $40^{\circ} \mathrm{C}$ in a Zymark Turbo Vap LV evaporator and redissolved in $100 \mathrm{nl}$ acetonitrile separately and respectively. Racemic and optically pure thalidomides and its derivatives $(0.1 \mathrm{mg} / \mathrm{l})$ were also mixed with $90 \mathrm{ml}$ filtered riverine waters; containing $1.0 \mathrm{~g}$ of riverine sediment separately and respectively; shaked 5 min and kept overnight for $24 \mathrm{~h}$. The sediments were removed by centrifugation and thalidomides and its derivatives were extracted using acetonitrile $(50 \mathrm{ml})$ by sonicator. The extracted thalidomides and its derivatives were dried under air at $40^{\circ} \mathrm{C}$ in a Zymark Turbo Vap LV evaporator and redissolved in $100 \mathrm{nl}$ acetonitriles separately and respectively. These acetonitrile solutions obtained from water and sediment samples were used to analyze chiral thalidomides and its derivatives using above-cited stereoselctive HPLC conditions.

\section{Dynamics and Kinetics}

To determine the dynamics of thalidomide and its derivatives, chiral adsorption studied were also carried out using riverine water and sediment. The effect of various parameters such as concentration of racemates, contact time, $\mathrm{pH}$, temperature, and particle size of riverine sediment was studied. The dynamics of these racemates between sediment and water was carried out using $0.01-$ $0.1 \mathrm{mg} / \mathrm{ml}$ concentrations, $5-120 \mathrm{~min}$ contact times, $2-10$, $20-40^{\circ} \mathrm{C}$ temperatures, $1-10 \mathrm{~g} / 1$ doses, and $0-75,75-150$, 150-210, 210-250, 250-300, 300-425 $\mu \mathrm{m}$ particle size. The experimental protocol was adopted and used as described elsewhere. ${ }^{23}$ The dynamics and kinetics of chiral thalidomide and its derivatives in natural conditions were also
TABLE 1. Retention times, capacity, separation, and resolution factors for chiral resolution of thalidomide and its derivatives in standard solutions

\begin{tabular}{lcccccc}
\hline Chiral compounds & $\begin{array}{c}t_{\mathrm{r} 1} \\
S-(-)\end{array}$ & $\begin{array}{c}t_{\mathrm{r} 2} \\
R-(+)\end{array}$ & $\begin{array}{c}k_{1} \\
S-(-)\end{array}$ & $\begin{array}{c}k_{2} \\
R-(+)\end{array}$ & $\alpha$ & $\mathrm{Rs}$ \\
\hline Thalidomide & 26.3 & 36.0 & 2.60 & 3.93 & 1.51 & 1.95 \\
3-Methyl thalidomide & 25.2 & 35.4 & 2.45 & 3.85 & 1.57 & 2.00 \\
3-Ethyl thalidomide & 22.8 & 28.3 & 2.26 & 3.05 & 1.35 & 1.00 \\
3-Butyl thalidomide & 20.0 & 25.1 & 2.08 & 2.86 & 1.38 & 1.00 \\
\hline
\end{tabular}

Experimental conditions: column, Ceramosphere RU-2; mobile phase, pure methanol; flow rate, $1.0 \mathrm{ml} / \mathrm{min}$; detection, $230 \mathrm{~nm}$; concentration, $0.1 \mathrm{mg} / \mathrm{ml}$; injected amount, $5.0 \mu \mathrm{l}$.

evaluated by using well-known models and equations. ${ }^{17}$ The concentrations of enantiomers of thalidomide and its derivatives in water and sediment samples were analyzed using above-cited SPE and HPLC conditions.

\section{RESULTS AND DISCUSSION Chiral Separations}

Chromatographic parameters such as capacity $(k)$, separation $(\alpha)$, and resolution (Rs) factors for the resolved enantiomers of thalidomide and its derivatives in standard solutions and riverine water and on sediment samples are given in Tables 1 and 2, respectively. A typical chromatogram of chiral separation of thalidomide under reported conditions is shown in Figure 1C. A perusal of Tables 1 and 2 clearly indicates that enantiomers of thalidomide and its derivatives were resolved successfully. The slight lower values of chromatographic parameters in riverine water and sediment may be due to interference of some other species present in the sediment. Retention times, capacity, separation, and resolution factors of the enantiomers of these drugs were in the range of 20.0-36.0, 2.08-3.93, 1.35-1.57, and 1.0-2.0 min, respectively. The order of the elution was confirmed by using optically active pure $R$-(+)-forms of each compound. It has been observed that $S$-(-)-enantiomer eluted first followed by $R$-(+)-enantiomer of all the studied racemates. A variation in the chromatographic parameters was carried out to obtain the best resolution. To optimize the chromatographic conditions, ethanol, acetonitrile, sodium perchlorate, and several buffers were tested but no good resolution could be achieved. Ethanol containing 0.1\% trifluoroacetic acid and triethylamine was also tested as the mobile phases but no good resolution could be achieved. As a result of extensive experimentation, the optimized chromatographic conditions were developed and reported herein.

\section{Chiral Separation at Supramolecular Level}

The supramolecular structure of the used CSP is shown in Figure 1B and it contains a spherical clay (sodium magnesium silicate) as the basic packing material with a ruthenium complex [(1,10-phenanthroline) ruthenium II complex, $\Delta$ configuration] as chiral selector. It has alternative sheets of octahedron of magnesium and tetrahedron of silica sandwiching chiral selector resulting in chiral cav- 
TABLE 2. Retention times, capacity, separation, and resolution factors for chiral resolution of thalidomide and its derivatives in river water and sediment

\begin{tabular}{|c|c|c|c|c|c|c|}
\hline Chiral compounds & $t_{\mathrm{r} 1} S-(-)$ & $t_{\mathrm{r} 2} R-(+)$ & $k_{1} S-(-)$ & $k_{2} R-(+)$ & $\alpha$ & Rs \\
\hline Thalidomide & $26.2(26.1)$ & $35.7(35.6)$ & $2.58(2.56)$ & $3.90(3.88)$ & $1.51(1.50)$ & $1.92(1.90)$ \\
\hline 3-Methyl thalidomide & $25.1(25.0)$ & $35.2(35.1)$ & $2.42(2.40)$ & $3.81(3.80)$ & $1.57(1.55)$ & $2.00(2.00)$ \\
\hline 3-Ethyl thalidomide & $22.6(22.0)$ & $28.1(28.0)$ & $2.24(2.22)$ & $3.01(3.00)$ & $1.35(1.33)$ & $1.00(1.00)$ \\
\hline 3-Butyl thalidomide & $19.8(19.5)$ & $25.0(25.0)$ & $2.06(2.02)$ & $2.82(2.80)$ & $1.37(1.35)$ & $1.00(1.00)$ \\
\hline
\end{tabular}

The values of chromatographic parameters inside and outside parenthesis correspond to water and sediment, respectively.

Experimental conditions: as in Table 1.

ities. Therefore, the tetrahedral and octahedral cavities having the ruthenium complex serve as chiral baskets and racemates can be separated once they enter these cavities. The exact chiral recognition mechanism on this phase is not known but it can be rationalized that the enantiomers form transient diasteromeric complexes with ruthenium complex, which are stabilized mainly by $\pi-\pi$ interactions with $\pi$ electron of phenanthroline ring system. Enantiomeric separation of these racemates is due to their penetration into the tetrahedron and octahedral cavities of silica and magnesium clay where they form transient diastereomeric complexes with chiral ruthenium complex which are stabilized by $\pi-\pi$ interactions between aromatic rings and 1,10-phenanthroline. Besides, other interactive forces such as steric, van der Waals forces may contribute toward the chiral resolution on the reported CSP. Briefly, aromatic ring of each enantiomers fits stereogenically in different fashion into chiral cavities of stationary phase which is stabilized by the $\pi-\pi$ interactions of different magnitude for both $R-(+)$ and $S$-(-)-enantiomers and, hence, the resolution of enantiomers occurred. However, stronger hydrogen bondings are formed between hydrogen atom, attached to $\mathrm{N}-\mathrm{H}$ amide group and oxygen atoms of clay. It is important to mention that the nature of hydrogen bonding is achiral, which binds the enantiomers strongly to the clay and, hence, enantiomers are eluted at high retention times. However, achiral hydrogen bondings provide sufficient time to the enantiomers to rest onto ruthenium complex, which may be helpful in chiral resolution.

\section{Solid Phase Extraction}

The SPE of thalidomides and its derivatives was optimized by adjusting different $\mathrm{pHs}$ of riverine water and flow rates. The flow rates of the eluting solvent (acetonitrile) were also varied. Besides, other eluting solvents such as ethanol, ethyl acetate, acetone, diethylether, chloroform, hexane, dichloromethane were also tried. As a result of exhaustive experimentation, the optimized SPE conditions were developed and used as mentioned in the experimental section. The percentage recoveries of thalidomide and its derivatives were good and ranged from 95.0 to $90.0 \%$ during dynamic equilibrium, kinetic and chiral interconversions experiments. It has also been observed that percentage recoveries of racemic and optically active thalidomides were slightly higher than those of its derivatives, which may be due to poor binding of laters with $\mathrm{C}_{18}$ material. The poor adsorption of thalidomide derivatives on reversed phase material of cartridges may be explained at Chirality DOI 10.1002/chir supramolecular level by considering steric effect of bulky alkyl groups present in thalidomide derivatives.

\section{Dynamics and Kinetics}

To understand the fate of thalidomide and its derivatives in nature, the dynamic equilibrium studies were carried out in riverine water-sediment system. The study involves adsorption on river bed sediment, dynamic equilibrium between riverine water and sediment, kinetics and chiral interconversions. The results of these experiments are discussed in the following sections.

\section{Equilibrium Dynamics}

The equilibrium and adsorption of thalidomide and its derivatives were carried out at different contact time, concentrations, doses, pHs, particle sizes, and temperatures. The effects of these parameters for thalidomide enantiomers only are shown in Figures 2A to 3A. These figures indicate that a dynamic equilibrium existed at $40 \mathrm{mg} / 1$ concentration with $8 \mathrm{~h}$ contact time. The equilibrium $\mathrm{pH}$ was 7.0 with $8 \mathrm{~g} / 1$ sediment dose. The effect of temperature on dynamic equilibrium was also studied and it was observed that the larger amount of thalidomide was on sediment at $40^{\circ} \mathrm{C}$ indicating endothermic nature of adsorption. Among various particle sizes studied, the maximum uptake was on $0-75 \mu \mathrm{m}$ size but its percentage is poor in riverine bed sediment and, hence, $210-250 \mu \mathrm{m}$ particle size was used to carry out all studies. Basically, dynamic equilibrium was studied for both $R$ - and $S$-enantiomers of thalidomide and it was observed that $S$-enantiomers adsorbed slightly higher on sediment in comparison to $R$-antipode.

To find out the mechanistic parameters associated with thalidomide and its derivatives, the results obtained from the above plots were analyzed by the well-known models of Langmuir and Freundlich involving the following equations.

$$
\frac{1}{q_{\mathrm{e}}}=\frac{1}{Q_{0}}+\frac{1}{b Q_{0} C_{\mathrm{e}}}
$$

where, $q_{\mathrm{e}}$ is the amount adsorbed $(\mathrm{mg} / \mathrm{g}), C_{\mathrm{e}}$ is the equilibrium concentration of the adsorbate $(\mathrm{mg} / \mathrm{L})$, and $Q_{0}$ and $b$ are the Langmuir constants related to maximum adsorption capacity and energy change in adsorption, respectively (Fig. 3B). Similarly, eq. 2 was used for Freundlich model.

$$
\log q_{\mathrm{e}}=\log K_{\mathrm{F}}+\frac{1}{n} \log C_{\mathrm{e}}
$$



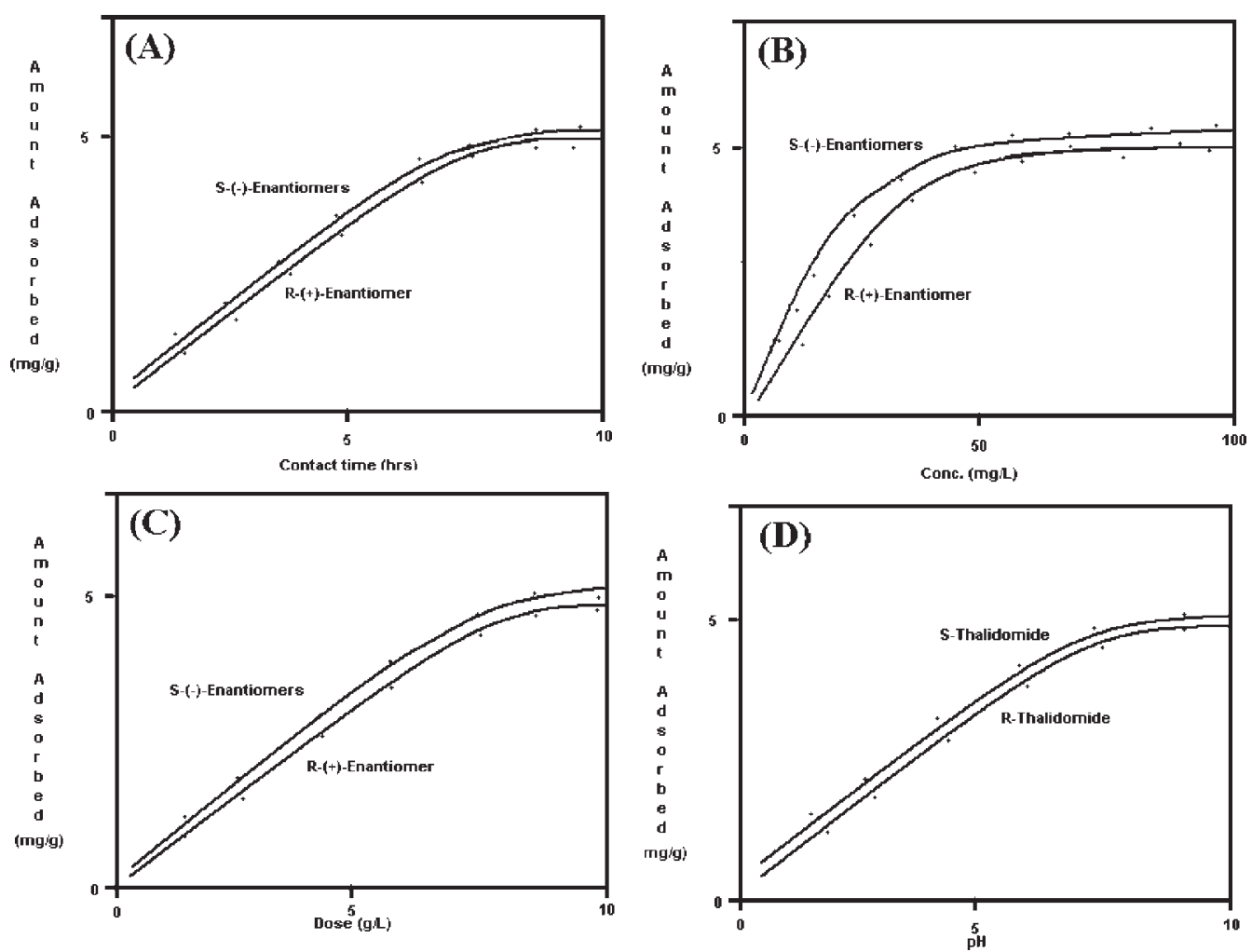

Fig. 2. Effect of (A) contact time, (B) concentrations, (C) dose, and (D) $\mathrm{pH}$ on uptake of $S$ - and $R$-thalidomide.
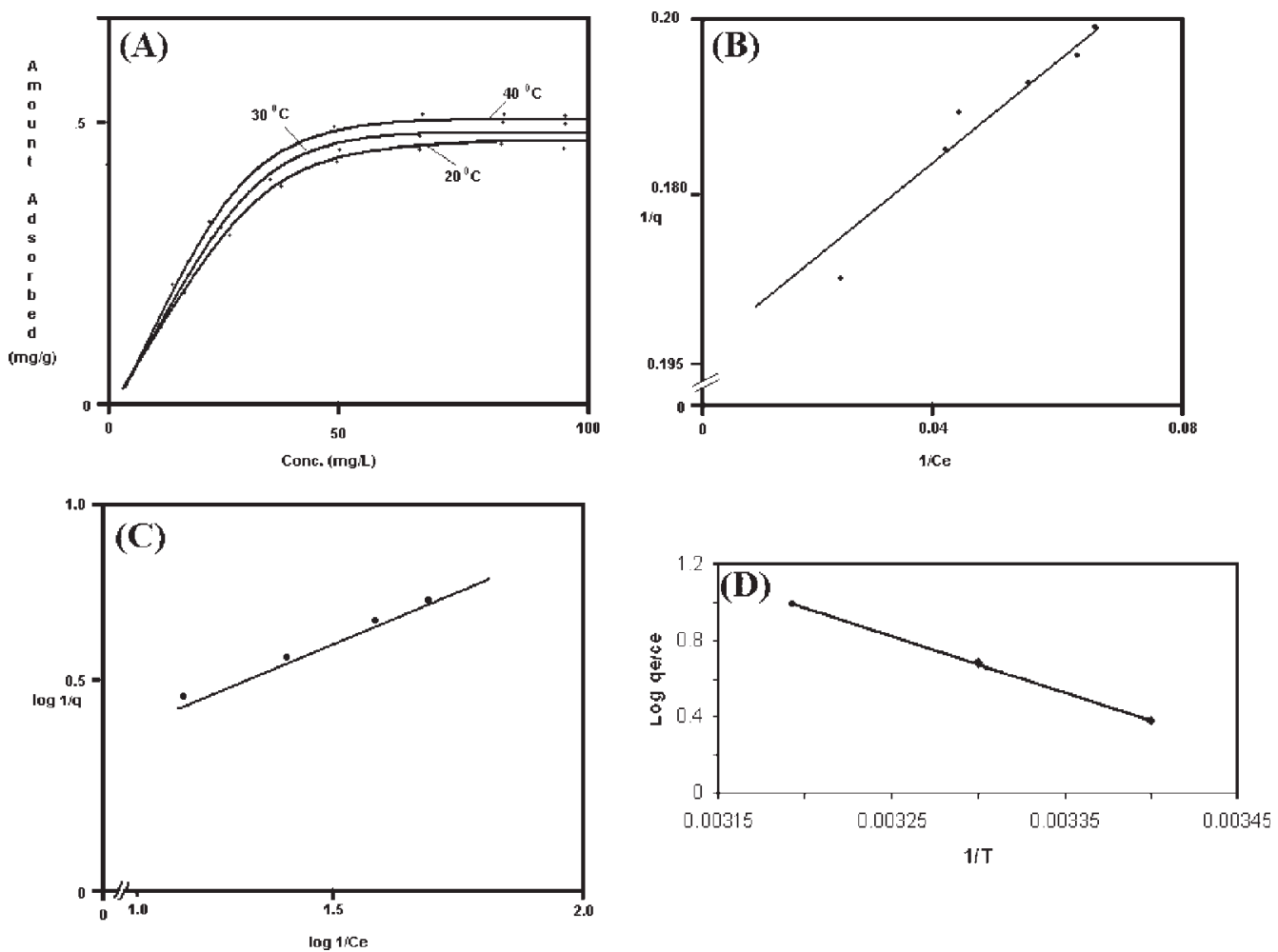

Fig. 3. Effect of (A) temperature on uptake of $S$-thalidomide, (B and C) Langmuir and Freundlich isotherms for $S$-thalidomide, and (D) plots of $\log \left(q_{\mathrm{e}} / C_{\mathrm{e}}\right)$ for adsorption of $S$-thalidomide on reverine sediment against $1 / T$ for different concentrations. 
TABLE 3. Langmuir constants of $S$-thalidomide and its derivatives

\begin{tabular}{|c|c|c|c|c|c|c|}
\hline \multirow[b]{2}{*}{ Compounds } & \multicolumn{3}{|c|}{$Q_{0}(\mathrm{mg} / \mathrm{g})$} & \multicolumn{3}{|c|}{$b(1 / \mathrm{mg})$} \\
\hline & $20^{\circ} \mathrm{C}$ & $30^{\circ} \mathrm{C}$ & $40^{\circ} \mathrm{C}$ & $20^{\circ} \mathrm{C}$ & $30^{\circ} \mathrm{C}$ & $40^{\circ} \mathrm{C}$ \\
\hline Thalidomide & 5.11 & 5.21 & 5.26 & $2.1 \times 10^{-3}$ & $1.95 \times 10^{-3}$ & $1.90 \times 10^{-3}$ \\
\hline 3-Methyl thalidomide & 5.00 & 5.11 & 5.15 & $2.0 \times 10^{-3}$ & $1.91 \times 10^{-3}$ & $1.88 \times 10^{-3}$ \\
\hline 3-Ethyl thalidomide & 4.91 & 4.96 & 5.00 & $1.92 \times 10^{-3}$ & $1.85 \times 10^{-3}$ & $1.80 \times 10^{-3}$ \\
\hline 3-Butyl thalidomide & 4.89 & 4.94 & 4.99 & $1.90 \times 10^{-3}$ & $1.82 \times 10^{-3}$ & $1.77 \times 10^{-3}$ \\
\hline
\end{tabular}

where, $q_{\mathrm{e}}$ is the amount adsorbed (mg/g), $C_{\mathrm{e}}$ is the equilibrium concentration of the adsorbate $(\mathrm{mg} / \mathrm{L})$ and $K_{\mathrm{F}}$ and $n$ are Freundlich constants related to the adsorption capacity and adsorption intensity, respectively (Fig. 3C). Langmuir and Freundlich constants are given in Tables 3 and 4, respectively. An evaluation of Figures $3 \mathrm{~B}$ and $3 \mathrm{C}$ and Tables 3 and 4 indicates the applicability and validity of the experiments conducted.

\section{Equilibrium Kinetics}

The kinetics of thalidomide and its derivatives in nature (riverine water-sediment system) was also studied by calculating free energy, enthalpy, and entropy. The changes in enthalpy $(\Delta H)$ and entropy $(\Delta S)$ were determined from the slope $(-\Delta H / 2.303 R)$ and intercept $(-\Delta S / 2.303 R)$ of the vant-Hoff's plot $\left[\ln \left(q_{\mathrm{e}} / C_{\mathrm{e}}\right)\right.$ vs. $\left.1 / T\right]$, respectively (Fig. $3 \mathrm{D}$ only for $S$-Thalidomide). The values of $\Delta H$ and $\Delta S$ for thalidomide derivatives were also calculated in the same way (figures are not given). The free energy change $(\Delta G)$ was calculated from the following equation:

$$
\Delta G=\Delta H-T \Delta S
$$

$$
\left.\log \left(q_{\mathrm{e}} / C_{\mathrm{e}}\right)=-\Delta H / 2.303 R T+\Delta S / 2.303 R\right)
$$

where $\left(q_{\mathrm{e}} / C_{\mathrm{e}}\right)$ is called the adsorption affinity and it is the ratio of $q_{\mathrm{e}}$, the amount adsorbed per unit mass at equilibrium to $C_{\mathrm{e}}$, the equilibrium concentration of the adsorbate. The thermodynamic parameters obtained for the adsorption of thalidomide and its derivatives under investigation are given in Table 5. Positive values of free energy indicate that the adsorption of thalidomide and its derivative is not spontaneous but rather slow.

The adsorption of thalidomide and its derivatives can be considered as a reversible reaction with equilibrium established between two phases. The Lagergren first-order rate eq. 6 can be applied for the determination of the adsorption rate constant.

$$
\log \left(q_{\mathrm{e}}-q_{t}\right)=\log q_{\mathrm{e}}-\frac{K_{\mathrm{ad}}}{2.303} t
$$

\begin{tabular}{|c|c|c|c|c|c|c|}
\hline \multirow[b]{2}{*}{ Compounds } & \multicolumn{3}{|c|}{$n$} & \multicolumn{3}{|c|}{$K_{\mathrm{F}}(\mathrm{mg} / \mathrm{g})$} \\
\hline & $20^{\circ} \mathrm{C}$ & $30^{\circ} \mathrm{C}$ & $40^{\circ} \mathrm{C}$ & $20^{\circ} \mathrm{C}$ & $30^{\circ} \mathrm{C}$ & $40^{\circ} \mathrm{C}$ \\
\hline Thalidomide & 6.25 & 6.30 & 6.34 & 4.47 & 4.50 & 4.55 \\
\hline 3-Methyl thalidomide & 6.20 & 6.25 & 6.30 & 4.45 & 4.48 & 4.52 \\
\hline 3-Ethyl thalidomide & 6.16 & 6.20 & 6.26 & 4.40 & 4.45 & 4.50 \\
\hline 3-Butyl thalidomide & 6.12 & 6.15 & 6.20 & 4.36 & 4.40 & 4.46 \\
\hline
\end{tabular}

TABLE 4. Freundlich constants of $S$-thalidomide and its derivatives

Chirality DOI 10.1002/chir where $q_{\mathrm{e}}$ and $q_{t}$ are concentrations of thalidomide and its derivatives at equilibrium and time $t$, and $K_{\mathrm{ad}}$ is the rate constant. Experimental data, measured at the optimal $\mathrm{pHs}$ for these molecules are plotted (figures are not given) and plots give straight lines with a slope $-K_{\mathrm{ad}} / 2.303$, confirming the applicability of the first-order Lagergren rate expression. Kinetic data were also analyzed by the procedure given by Reichenberg ${ }^{18}$ using eqs. 7-10.

$$
\begin{gathered}
F=1-\frac{6}{\pi^{2}} \sum_{n=0}^{n=n} \frac{1}{n^{2}} \exp \left[\frac{-D_{i} t \pi^{2} n^{2}}{r_{0}^{2}}\right] \\
\text { Or } F=1-\frac{6}{\pi^{2}} \sum_{n=0}^{n=n} \frac{1}{n^{2}} \exp \left[-n^{2} B t\right] \\
F=\frac{Q_{t}}{Q^{0}} \\
B=\frac{\pi^{2} D_{\mathrm{i}}}{r_{0}^{2}}
\end{gathered}
$$

where $F$ is the fractional attainment of equilibrium at time $t, Q_{t}$ is the amount of adsorbent taken up at time $t$ and $Q^{0}$ is the maximum equilibrium uptake (at infinite time), $D_{\mathrm{i}}$ is the effective diffusion coefficient of these molecules in the adsorbent phase, $B$ is the time constant, $r_{0}$ is the radius of the adsorbent particle (sediment), assumed to be spherical, and $n$ is an integer that defines the infinite series solution.

The $B t$ values were obtained for each observed value of $F$, from Reichenberg's eq. 8. The linearity test of $B t$ versus time plots was used to distinguish between the film diffusion and particle-diffusion-controlled adsorption.

\begin{tabular}{|c|c|c|c|c|c|}
\hline \multirow[b]{2}{*}{ Compound } & \multicolumn{3}{|c|}{$\Delta G^{\circ}(\mathrm{kJ} / \mathrm{K} \mathrm{mol})$} & \multirow{2}{*}{$\begin{array}{c}\Delta H^{\circ} \\
(\mathrm{kJ} / \mathrm{K} \mathrm{mol})\end{array}$} & \multirow{2}{*}{$\begin{array}{c}\Delta S^{\circ} \\
(\mathrm{kJ} / \mathrm{mol})\end{array}$} \\
\hline & $20^{\circ}$ & $30^{\circ}$ & $40^{\circ}$ & & \\
\hline Thalidomide & 1.83 & 3.82 & 5.81 & 56.48 & 0.20 \\
\hline 3-Methyl thalidomide & 1.85 & 3.85 & 5.90 & 50.89 & 0.22 \\
\hline 3-Ethyl thalidomide & 1.90 & 3.91 & 5.98 & 47.04 & 0.24 \\
\hline 3-Butyl thalidomide & 1.96 & 3.95 & 6.09 & 45.10 & 0.28 \\
\hline
\end{tabular}
If the plot of $B t$ vs. time (having slope $B$ ) is a straight line passing through the origin the adsorption rate is governed by the particle diffusion mechanism; otherwise, it is governed by film diffusion. For all drugs the plots are

TABLE 5. Equilibrium thermodynamic parameters for $S$-thalidomide and its derivatives 

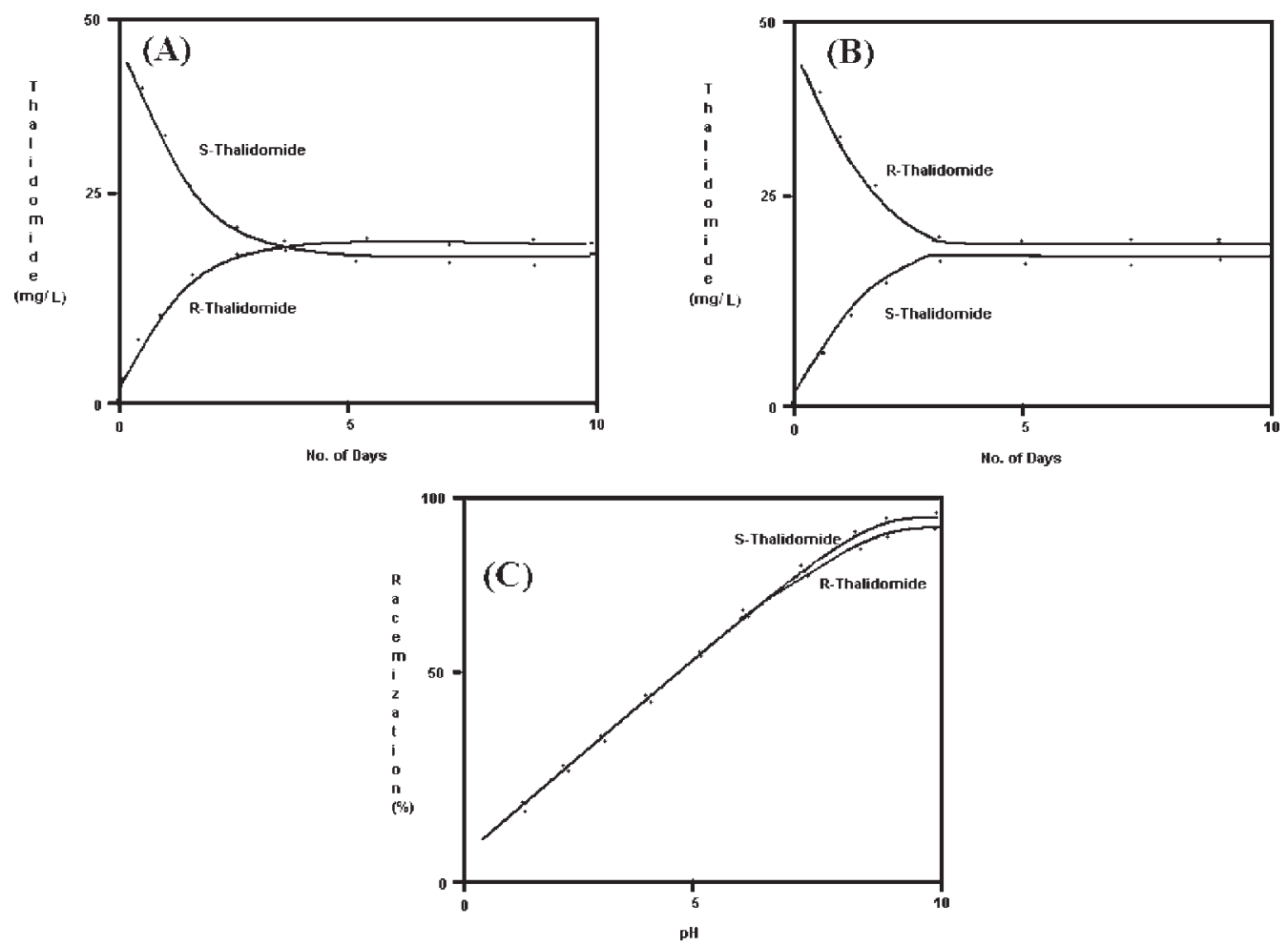

Fig. 4. Chiral interconversion of (A) $S$-thalidomide and (B) $R$-thalidomide enantiomers in water-sediment systems and the effect of $\mathrm{pH}$ on the chiral interconversions of $S$ - and $R$-thalidomide.

linear but do not pass through the origin indicating a film diffusion mechanism.

\section{Chiral Interconversions}

The chiral interconversions of $R$ - and $S$-thalidomide were studied under natural conditions. The solubilities of thalidomide and its derivatives in water are poor and, hence, the probability of their occurrence on sediment is high. Therefore, the chiral interconversions were studied on sediment after their adsorption on it. Optically active pure enantiomers of thalidomide and its derivatives were used for chiral interconversions. The chiral interconversions of $S$ - and $R$-thalidomide are shown in Figures $4 \mathrm{~A}$ and $4 \mathrm{~B}$ and the rate constants of the chiral interconversions were calculated by the following equation.

$$
k=(2.303 / t) \log a /(a-X)
$$

where $t$ is the time in days in which $x$ amount of racemate is formed from initial concentration $(a)$ of pure enantio- mers. The values of rate constants of the chiral interconversions for thalidomide and its derivatives are given in Table 6, which indicates higher rate of chiral interconversions of $S$-enantiomers of thalidomide and its derivatives. The values of rate constants were 0.390 and $0.385 \mathrm{day}^{-1}$ for $S$ - and $R$-enantiomers respectively, indicating slightly higher tendency of racemization of $S$-thalidomide. The effects of $\mathrm{pH}$ and temperature were studied on the chiral interconversions of thalidomide enantiomers. $\mathrm{pH}$ was varied from 2.0 to 10 and the results are shown in Figure 4C, which shows that racemization was higher at high $\mathrm{pH}$ (up to 9.0) and becomes constant after this $\mathrm{pH}$. The effect of temperature was carried out at three different values, i.e., 20,30 , and $40^{\circ} \mathrm{C}$ and slightly higher rate of chiral interconversions was observed at $40^{\circ} \mathrm{C}$.

\section{Mechanism of Dynamics at Supramolecular Level}

To ascertain the toxicities of thalidomide and its derivatives, it is important to understand the mechanism of dynamic equilibrium of thalidomide and its derivatives

TABLE 6. Rate constants $\left(\right.$ day $\left.^{-1}\right)$ of chiral interconversions of thalidomide and its derivatives at $30^{\circ} \mathrm{C}$ at in water of pH 7.5

\begin{tabular}{llr}
\hline Compounds & $S$-Thalidomide & $R$-Thalidomide \\
\hline Thalidomide & $1.88 /$ day $\left(2.17 \times 10^{-5} /\right.$ second $)$ & $1.84 /$ day $\left(2.13 \times 10^{-5} /\right.$ second $)$ \\
3-Methyl thalidomide & $1.03 /$ day $\left(1.19 \times 10^{-5} /\right.$ second $)$ & $1.00 /$ day $\left(1.15 \times 10^{-5} /\right.$ second $)$ \\
3-Ethyl thalidomide & 0.99/day $\left(1.14 \times 10^{-5} /\right.$ second $)$ & $0.95 /$ day $\left(1.10 \times 10^{-5} /\right.$ second $)$ \\
3-Butyl thalidomide & 0.94/day $\left(1.06 \times 10^{-5} /\right.$ second $)$ & $0.90 /$ day $\left(1.04 \times 10^{-5} /\right.$ second $)$
\end{tabular}


TABLE 7. Regression analysis data for chromatographic and SPE methods of thalidomide and its derivatives

\begin{tabular}{lccccccc}
\hline & \multicolumn{3}{c}{ HPLC (peak areas) } & & \multicolumn{2}{c}{ SPE (\% recoveries) } \\
\cline { 2 - 3 } \cline { 7 - 9 } Chiral compounds & SD & CC & CL & & SD & CC & CL \\
\hline Thalidomide & \pm 0.10 & 0.9998 & 99.5 & & \pm 0.05 & 0.9999 & 99.5 \\
3-Methyl thalidomide & \pm 0.10 & 0.9998 & 99.4 & & \pm 0.05 & 0.9999 & 99.4 \\
3-Ethyl thalidomide & \pm 0.10 & 0.9998 & 99.5 & & \pm 0.05 & 0.9999 & 99.5 \\
3-Butyl thalidomide & \pm 0.10 & 0.9999 & 99.4 & & \pm 0.05 & 0.9999 & 99.4 \\
\hline
\end{tabular}

SD, standard deviation; CC, correlation coefficients; CL, confidence limit.

under natural conditions. $\mathrm{p} K_{\mathrm{a}}$ values of thalidomide and its derivatives are more than 10.0 and, hence, these drugs exist as neutral molecules at the experimental conditions reported in this work. The dynamic equilibrium was found to be film diffusion at supramolecular level and is controlled by certain forces such as van der Waal, hydrogen bondings, steric effects. These drugs contain electronegative atoms such as oxygen and nitrogen and on the other hand sediment has hydroxyl and silicon groups. These groups and atoms form hydrogen bondings between these drugs and sediment. The adsorption of these drugs is endothermic indicating a slow process at natural conditions. The order of adsorption is thalidomide $>3$-methyl thalidomide $>$ 3-ethyl thalidomide $>3$-butyl thalidomide, indicating the role of the steric effects of the alkyl groups, i.e., methyl, ethyl, and butyl in thalidomide derivatives; in comparison to thalidomide. In summary, various physical forces and the structures of these drugs at supramolecular level are responsible for different dynamic equilibrium concentrations of the studied racemates under the reported experimental conditions. Both thalidomide enantiomers are not stable under natural conditions and, hence get racemized. The chiral interconversions is favored at low $\mathrm{pH}$ indicating a mechanism through proton exchange phenomenon as discussed by other authors. ${ }^{15-18,24,25-28}$

\section{VALIDATION}

Validation of the developed methods was ascertained by carrying out five sets $(n=5)$ of the chromatographic and SPE procedures under identical conditions. The regression analysis was carried out using Microsoft Excel program and the results are given in Table 7 for chromatographic and SPE procedures, respectively. Table 7 shows that standard deviation was \pm 0.10 and \pm 0.05 for chromatographic and SPE methods while the correlation coefficients $\left(R^{2}\right)$ and confidence levels were $0.9999-0.9998 \%$ and $99.4-99.5 \%$, respectively.

\section{CONCLUSIONS}

The presence of thalidomide and its derivatives in nature is hazardous and this study indicates that most of these drugs remain on sediment with poor concentration in aqueous phase. Sediment has the capacity to hold these toxic drugs and, hence, we are thankful to nature for its self purification power. However, there is a limit of the Chirality DOI 10.1002/chir sediment to hold these drugs when present at high concentration (more than $40 \mathrm{mg} / \mathrm{l}$ ), consequently, thalidomide and its derivatives are supposed to be present in water, which can enter into human body. The chiral interconversions of the safe $R$-enantiomers to the $S$-toxic enantiomer under natural condition is a serious phenomenon. The present work describes the dynamics of the chiral thalidomide at supramolecular level, in natural conditions. This study may be useful to control the contamination of earth ecosystem because of these toxic drugs. Therefore, one should be careful before releasing waste effluents, containing thalidomide, in the nature. Such type of effluent discharges should be treated properly before their release into the environment.

\section{LITERATURE CITED}

1. McBride W. Thalidomide and congenital abnormalities. Lancet 1961; 2:1398-1400.

2. Mellin G, Katzenstein MN. The saga of thalidomide. Neuropathy to embryopathy, with case reports of congenital anomalies. N Engl J Med 1962;267:1184-1193.

3. Blaschke G, Kraft HP, Fickentscher K, Köhler F. Chromatographic separation of racemic thalidomide and teratogenic activity of its enantiomers. Arzneimittel Forsch. ( Drug Res.) 1979;29:1640-1642.

4. Eriksson T, Bjorkman S, Hoglund P. Clinical pharmacology of thalidomide. Eur J Clin Pharmacol 2001;57:365-376.

5. Sleijfer S, Kruit WH, Stoter G. Thalidomide in solid tumours: the resurrection of an old drug. Eur J Cancer 2004;40:2377-2382.

6. Jakeman P, Smith WCS. Thalidomide in leprosy reaction. Lancet 1994;343:432-433.

7. Shimazawa R, Miyachi H, Takayama H, Kuroda K, Kato F, Kato M, Hashimoto Y. Antiangiogenic activity of tumor necrosis factor-alpha production regulators derived from thalidomide. Biol Pharm Bull 1999;22:224-226.

8. Makonkawkeyoons S, Limson-Pobre RNR, Moreira AL, Kaplan G. Thalidomide inhibits the replication of human immunodeficiency virus type 1. Proc Natl Acad Sci USA 1993;90:5974-5978.

9. Chuong PH, Galons H, Voisin J, Righenzi S, Warnet JM, Claude JR, Huynh-Thien D. In vitro and in vivo immunosuppressive potential of thalidomide and its derivative, N-hydroxythalidomide, alone and in combination with cyclosporin A. Int J Immunopharmacol 1997;19:289-296.

10. Bernstein JR. Thalidomide. Clin Toxicol Rev 1999;21:5.

11. Georghiou PR, Allworth AM. Pasteurella multocida infection after a Tasmanian devil bite. Clin J Infect Dis 1992;166:939-940.

12. Gunzler V. Thalidomide in human immunodeficiency virus (HIV) patients. A review of safety considerations. Drug Saf 1992;7:116-134.

13. Vogelsang GB, Farmer ER, Hess AD, Altamonte V, Beschorner WE, Jabs DA, Corio RL, Levin LS, Colvin OM, Wingard JR, Santos GN. Thalidomide for the treatment of chronic graft-versus-host disease. $\mathrm{N}$ Engl J Med 1992;326:1055-1058.

14. Youle M, Clarbour J, Farthing C, Connolly M, Hawkins D, Staughton R, Gazzard B. Treatment of resistant aphthous ulceration with thalidomide in patients positive for HIV antibody. Br Med J 1989;298:432-436.

15. Testa B, Carrupt PA, Gal J. The so-called interconversion of stereoisomeric drugs: an attempt at clarification. Chirality 1993;5:105-111.

16. Knoche B, Blaschke G. Investigations on the in vitro racemization of thalidomide by high-performance liquid chromatography. J Chromatogr A 1994;666:235-240.

17. Knoche B, Blaschke G. Stereoelectivity of the in vitro metabolism of thalidomide. Chirality 1994;6:221-224.

18. Eriksson T, Bjrkman S, Roth B, Fyge A, Hglund P. Stereospecific determination, chiral inversion in vitro and pharmakokinetics in humans of the enantiomers of thalidomide. Chirality 1995;7:44-52.

19. Weinz C, Blaschke G. Investigation of the in vitro biotransformation and simultaneous enantioselective separation of thalidomide and its 
neutral metabolites by capillary electrophoresis. J Chromatogr B 1995;647:287-292.

20. Eriksson T, Bjorkman S, Roth B, Fyge A, Hoglun P. Enantiomers of thalidomide: blood distribution and the influence of serum albumin on chiral inversion and hydrolysis. Chirality 1998;10:223-228.

21. Reist M, Carrupt PA, Francotte E, Testa B. Chiral inversion and hydrolysis of thalidomide: mechanisms and catalysis by bases and serum albumin and chiral stability of teratogenic metabolites. Chem Res Toxicol 1998;11:1521-1528.

22. Meyring M, Mhlenbrock C, Blaschke G. Investigation of the stereoselective in vitro biotransformation of thalidomide using a dual cyclodextrin system in capillary electrophoresis. Electrophoresis 2000;21: 3270-3279.

23. Gupta VK, Ali I. Removal of endosulfan and methoxychlor from water on carbon slurry. Environ Sci Technol 2008;42:766-770.
24. Gupta VK, Ali I, Saini VK. Removal of chlorophenols from wastewater using red mud: an aluminum industry waste. Environ Sci Technol 2004;38:4012-4018.

25. Reichenberg D. Properties of ion exchange resins in relation to their structure. III. Kinetics of exchange. J Am Chem Soc 1953;75: 589-597.

26. Trapp O, Schoetz G. Schurig V. Stereointegrity of thalidomide: gas chromatographic determination of the enantiomerization barrier. J Pharam Biomed Anal 2002;27:497-505.

27. Schoetz G, Trapp O. Schurig V. Determination of the enantiomerization barrier of thalidomide by dynamic elelctrokinetic chromatograpphy. Electrophoresis 2001;22:3185-3190.

28. Trapp $\mathrm{O}$. The unified equation for the evaluation of degenerated frist order reactions in dynamic electrophoresis. Electrophoresis 2006;27: 2999-3006. 